\title{
Frontières
}

\section{Destin de la norme}

\section{Christian Saint-Germain}

Volume 14, numéro 1, automne 2001

Où est la marge ?

URI : https://id.erudit.org/iderudit/1074164ar

DOI : https://doi.org/10.7202/1074164ar

Aller au sommaire du numéro

Éditeur(s)

Université du Québec à Montréal

ISSN

1180-3479 (imprimé)

1916-0976 (numérique)

Découvrir la revue

Citer ce document

Saint-Germain, C. (2001). Destin de la norme. Frontières, 14(1), 63-66.

https://doi.org/10.7202/1074164ar

Ce document est protégé par la loi sur le droit d'auteur. L'utilisation des services d'Érudit (y compris la reproduction) est assujettie à sa politique d'utilisation que vous pouvez consulter en ligne.

https://apropos.erudit.org/fr/usagers/politique-dutilisation/
Cet article est diffusé et préservé par Érudit.

Érudit est un consortium interuniversitaire sans but lucratif composé de l’Université de Montréal, l'Université Laval et l'Université du Québec à Montréal. Il a pour mission la promotion et la valorisation de la recherche. https://www.erudit.org/fr/ 


$\begin{array}{llllllllll}P & O & \text { I } & \text { N } & \text { T } & \text { D } & \text { E } & \text { V } & \text { U } & \text { E }\end{array}$

\section{DESTIN \\ DE LA NORME}

« CETTE DÉMOCRATIE SI PARFAITE FABRIQUE ELLE-MÊME SON INCONCEVABLE ENNEMI, LE TERRORISME.

ELLE VEUT, EN EFFET, ÊTRE JUGÉE SUR SES ENNEMIS PLUTÔT QUE SUR SES RÉSULTATS. LES POPULATIONS SPECTATRICES

NE PEUVENT CERTES PAS TOUT SAVOIR DU TERRORISME, MAIS ELLES PEUVENT TOUJOURS EN SAVOIR ASSEZ POUR ÊTRE PERSUADÉES QUE, PAR RAPPORT À CE TERRORISME, TOUT LE RESTE DEVRA LEUR SEMBLER PLUTÔT ACCEPTABLE, EN TOUT CAS PLUS RATIONNEL ET PLUS DÉMOCRATIQUE. "

DEBORD, 1992, p. 40

\section{Christian Saint-Germain, Ph.D., professeur titulaire \\ au Département de philosophie de I'UQAM.}

Le concept de marginalité ne répond plus. Dans la modernité, cette référence à une marge n'a toujours eu pour fonction que de rassurer quant à l'existence présumée d'une page sociale. Or, rien n'est pourtant désormais moins sûr, car il n'y a plus d'écart minoritaire qui ne soit détourné de ses fins. Il faudrait pouvoir décrire l'assemblage des sociétés de consommation actuelles comme un «en-semble» ou un simulacre permanent du vivre en commun. À l'époque du bonheur planifié (nous avons inventé le bonheur disait le dernier homme de Nietzsche), il en va des individus, des consommateurs, comme autant d'atomes à faire circuler dans un dispositif de décisions d'achats prédictibles. Il s'agit de produire l'individualisme non comme accès à la singularité mais uniformisation des consciences. Les critères de marginalité ou de normalité sont alors en chute libre, en perte de signification massive.

Nous souhaiterions suggérer la disparition progressive, voire l'inutilité du concept de marginalité pour décrire la vie sociale. La capacité de résister à la domination d'un modèle économique unitaire, «intégriste», ayant été entièrement dissipé. Plus exactement, les diverses formes de la contestation ne peuvent désormais, à défaut ou en l'absence de modèle substitutif du vivre en commun, que tourner à la parodie : participer de manière équivoque et collaborer finalement avec l'industrie du spectacle, de l'information.

Le problème des sociétés modernes, comme sociétés démocratiques, est qu'elles tendent à transformer en un écheveau toujours plus inextricable la relation complexe entre la situation normale, la constitution normative de l'Ordre, l'état de droit et l'exception, l'événement, l'acte souverain. Elles rendent toujours plus difficilement praticable la distinction entre l'inclus et l'exclu, le dedans et le dehors et du coup tous les partages organisationnels 
qui s'y réfèrent - le civilisé et le

barbare, le normal et le pathologique,

l'autochtone et l'étranger, le familier et

l'inquiétant, le même et l'autre sous les espèces, par exemple, du "petit homme» et du monstre. (Brossat, 1996, p. 185)

Le statut éphémère «d'autre » au magma consumériste est celui conféré par la publicité telle qu'elle peut apparaître chez Benetton, délibérément agressive comme subterfuge de captage d'attention pour écouler des produits ou en signaler l'existence.

\section{LE « DÉSENCRAGE » DES MARGES}

Le texte social ne se laisse plus guère lire qu'en diagonale. Les petites balises d'encre qui tiennent en état une page et ses interlignes s'évaporent. Cela ne laisse pourtant aucune place à une écriture nouvelle, à d'autres dessins, mais à la disposition de minces gargouilles éphémères, à l'inscription sur les murs de graffiti. Pauvre expression qui rappelle qu'il n'y a jamais eu autant de murs entre les êtres, d'invisibles barbelés qui traversent notre condition historique de consommateur définitif.

Les frontières sont désormais flottantes entre la normalité et la marginalité. Une incertitude sans précédent pèse sur les jugements normatifs tant par une désensibilisation que par un «désapprentissage» $\mathrm{du}$ jugement moral des individus. Cette situation anomique sonne le glas du concept de marginalité. Bien sûr, personne ne nie l'existence des populations disqualifiées économiquement, des minorités: malades mentaux, drogués, assistés sociaux, jeunes gens surnuméraires acceptant une succession d'emplois absurdes. Rien toutefois qui ne soit susceptible d'opposer la moindre résistance à l'écrasante transparence et à l'acceptation tacite de la domination économique. En fait, il ne reste de la marginalité que parce que ce concept ne survit qu'assisté lui-même par des institutions de spécialistes de ces anciennes catégories sociales : travailleurs sociaux, intervenants de tout acabit, cortège d'avatars pastoraux ou de préposés de la bonne conscience.

Il reste des minorités mais pas au sens où celles-ci s'opposeraient à une majorité restante. Subsistent plutôt des groupes autoproclamés minoritaires comme il existe des études féministes, des études gaies et lesbiennes et que se construisent des carrières intellectuelles bien tranquilles sur simple évocation du désavantage numérique ou de l'aliénation historique mondiale. Il ne reste qu'une expérience «monumentale» ou mieux «muséologique » de ces rapports de force dans la société. Une sorte de parodie instituée du changement. Il faut dénoncer à cet égard la confusion abusive entre la tolérance véritable d'autrui et l'indifférence vécue désormais à puissance exponentielle.
De fait, s'il fallait conserver l'idée d'une marginalité ou de marge, il faudrait savoir la penser désarrimée, une bribe de texte vif arraché et constitué d'un alphabet explosif.

Dans l'espace social actuel, le rapport à la détresse d'autrui ne se pense que sous le couvert de considérations hygiéniques ou sanitaires tant le rapport à la conscience morale d'un soi social consistant s'est évanoui. Ainsi voit-on suggérés la distribution de seringues gratuites, l'établissement de " piqueries supervisées », de groupes compassionnels pour la légalisation des drogues douces, sans se douter que cette apparente incitation à l'élargissement du cadre légal cache son moment de réversibilité répressive. La marijuana s'avérera, une fois acceptée collectivement, un puissant outil de refroidissement des conduites contestataires. À vrai dire, il ne fait aucun doute que ce moyen d'évasion sera non seulement légalisé mais géré et taxé par l'État, de la même manière que ce dernier s'est substitué au monde interlope pour s'assurer la gestion exclusive des jeux et des paris. La consommation de drogues douces par les citoyens représente un moyen peu coûteux et efficace de contrôle des populations éventuellement récalcitrantes. Au même titre que l'apparent progrès du droit à l'avortement débouche, non d'une manière causale mais incidente, sur la disponibilité de «matériel » humain pour l'expérimentation. C'est le modèle de la gestion concentrationnaire qui est décisif pour comprendre le déploiement de la politique occidentale. Exprimé en termes choquants: échangerions toxicomanes en voie de réhabilitation contre vieillards en perte d'autonomie pour notre centre de jour.

\section{MÉDICALISATION}

\section{DU PARC HUMAIN}

C'est que le politique n'est plus un enjeu suffisamment attirant pour commander quelque vigilance du citoyen, si l'on dispose cette préoccupation pourtant essentielle sur une échelle d'intérêts immédiats. Ce n'est d'ailleurs qu'à partir de cette échelle que l'univers des individus organise sa gravité propre. Dans un espace dense de conditionnement économique et de divertissement planétaire, l'objectif des gouvernants reste celui de développer des stratégies pour obtenir l'attention des sujets. Cette politique intensive de sollicitation mène le plus souvent à la mise en place de moyens extrêmement vulgaires d'une pédagogie des masses, qui vont du voyeurisme de la vie ordinaire des autres, en passant par la publicité étatique sur la nécessité d'utiliser des condoms. Ce souci sanitaire rapproche le parc humain du cheptel d'élevage de bovins à qui l'on insère continûment des antibiotiques dans les nourritures pour éviter les épidémies ou augmenter le rendement en protéines sur les carcasses à l'abattage. Il importe d'exproprier l'intime, de mettre à la vue, en pure transparence de dépossession réciproque la vie du citoyen. Il suffit à cet égard qu'un photographe invite au centre-ville deux milles inconnus à se dévêtir pour que surgisse, comme dans un charnier imaginaire, une palette de corps livides, tous sillons confondus. Est-ce la contrepartie du charnier invisible de l'Étatprovidence prenant en charge les corps à partir de la naissance planifiée et monitorée pendant la grossesse jusqu'au combat «épique» contre l'ostéoporose, le vieillissement prématuré et finalement l'abandon administratif en centre d'accueil ? Ne reste$\mathrm{t}$-il en Occident du politique que la figure martiale des combats contre les maladies, la mort? Une politique de survie de soi à tout prix?

La quête sociale de l'attention n'est cependant aucunement en contradiction avec son pôle opposé selon lequel tout a déjà été vu et se répète : une oreille en peau humaine sur le dos d'une souris vivante, une grand-mère inséminée, la découverte d'une fosse commune au Kosovo ou en Birmanie, etc. Le format rend déjà banale, comme par anticipation, la possibilité d'indignation: c'est déjà arrivé, que peuton encore y faire? Ou bien, cela se prépare, il est trop tard pour empêcher quoi que ce soit. Il n'est d'autre option que de continuer à vivre dans le souci suburbain d'autres images portées par les flots d'événements interchangeables, de catastrophes de remplacement.

Dans ce contexte, il n'est plus possible de concevoir la moindre résistance à une structure de domination économique à ce point intériorisée, il n'est point d'issues collectives perceptibles. L'univers se pose comme une grande surface, marché aux espèces, aux vivants, ou encore aux esclaves dans le cas de la confection des produits importés de consommation courante.

\section{CONTESTATION RIDICULE ET CONTESTATION RADICALE}

Un fait divers ponctuel pourrait éclairer cette esquisse descriptive. Des jeunes gens venus en autobus scolaires lancent des pierres sur des policiers casqués et armés. Un périmètre bien statique et visible est déployé. Un attroupement d'hommes en armes avec moult renfort de gaz, d'eau sous pression et de bâtons attendent les débordements inlassablement évoqués par les médias. Un quartier de la ville de Québec, sous les gaz lacrymogènes, n'a pas autant pleuré depuis la défaite de Montcalm. Le «spectacle » des antagonismes n'a pas déçu. Mille fois repris à la télévision avec en prime : des commentateurs larmoyants sur 
fond d'esbroufe, pour les fins du réalisme mais surtout le déjà-vu des sommets, la puissante force d'indifférence de cette répétition d'images creuses.

La formation à la résistance passive n'aura apparemment pas porté des fruits. Les médias filmaient préalablement à cette rencontre les techniques militantes enseignées pour se faire arrêter en s'enchaînant mutuellement et en acceptant de laisser traîner un à un vers les paniers à salade tous les fessiers de denim disponibles. Autre leçon: comment se rincer du poivre de Cayenne désormais au menu pour les figurants à ces rencontres. Cette soumission à la violence policière, le fait de se présenter très précisément là où l'on est attendu ravalent cet exercice à une contestation bien modeste. Une résistance inédite reste à inventer, insidieuse, irrécupérable, une expression qui échapperait à sa reproduction médiatique, à son incarcération picturale.

Les événements médiatiques que constitue la description des sommets économiques frappent par leur insignifiance. Plus exactement par la disproportion des moyens pour mettre en ondes, en scène, les actes de vandalisme et la signification sociale dont ils sont apparemment les porteurs: malaise devant la mondialisation, emprise grandissante de l'économie, etc. Ces actes de désobéissance temporaire ne sont de fait que d'agréables moments du divertissement planétaire, dût-il arriver à l'occasion de ces scénarios qu'un homme meure sous les balles de caoutchouc ou de fer. Au cinéma, il arrive aussi que des accidents se produisent entre cascadeurs ou figurants. Résister à une idéologie dominante ne consiste pas à se porter à la rencontre d'un dispositif policier organisé et statique. Seuls les promoteurs des combats de boxe procéderaient à une pareille stratégie publicitaire.

À cet égard, la mise en scène récente du fonctionnement démocratique n'est pas sans procurer à l'observateur un inconfortable amusement. Alors que la plupart des sociétés développées sont contrôlées de manière abstraite et invisible, le citoyen manifeste encore rituellement sa désapprobation ponctuelle et verse, sous sollicitude de gaz lacrymogènes, des larmes de crocodiles démocratiques. Cette passivité sous les gaz, l'acquiescement partiel à la répression par collision chorégraphiée à un dispositif policier statique constituent la même illusion avec laquelle se bercent et finalement s'endorment les groupes. Comme si en descendant dans la rue ou en allant déposer un bulletin de vote, la seule manifestation d'indignation ou de mécontentement allait faire contrepoids aux intérêts solidement ancrés d'autres masses abstraites. Se faire entendre est une chose, se faire écouter en est une autre. Imaginons une dernière fois la scène sous la forme d'une demande expresse : auriez-vous l'obligeance, représentants du capital spéculatif, d'interrompre le versement des plantureux dividendes au cercle restreint de vos actionnaires sans quoi nous déserterons nos téléviseurs et nos centres d'achats...?

C'est qu'il n'en va pas de forces de même nature sur la ligne d'engagement. Il faudrait pour exercer au sens physique quelque résistance, développer une force égale à celle de l'avidité du déforesteur ou du cloneur à gages. Mais tenter d'éconduire par une énergie suffisante ces intérêts par la simple expression de l'opinion contraire ressemble au battement d'un éventail de soie que l'on voudrait proportionnelle à la force ordonnée d'une rangée de pistons.

\section{GUERRE DE RELIGION ET D'ÉCONOMIE}

Plus sérieusement cette fois, l'effondrement des plus orgueilleuses tours du commerce mondial montre la fragilité de ce mode de vie, de cette désinvolture individuelle à l'égard du politique. Comme s'il fallait réaliser par la manière forte la fragilité de ce qui paraît aller de soi. Il n'est pas d'innocence possible à notre être de consommateur. Se rendre à son travail le matin pour participer au monde du commerce reste inscrit dans une politique de la violence, comme le faisaient déjà d'honnêtes chefs de famille allant, pendant la guerre du Vietnam, participer à la fabrication syndiquée du napalm ou des défoliants. Une fois les larmes séchées et les caméras éteintes, l'événement s'avère un puissant révélateur "photographique » quant à notre degré d'ignorance à l'égard de la fermeté de la conscience religieuse. Il semble que tous ne participent pas encore à notre engouement pour l'allongement de la vie biologique et pour l'espérance, toute pharmaceutique, de vie du consommateur moyen.

Il ne viendrait à personne au Québec l'idée que la religion puisse encore galvaniser des populations jeunes jusqu'au sacrifice. Ici, une assemblée de vieillards se réunit sans conviction autour d'oripeaux homilétiques. Nous restons captifs de notre image d'une religion vécue sous le mode du buffet à numéro. Un peu de bouddhisme, du christianisme pour les mariages et les enterrements, de la réincarnation de-ci de-là. Autant dire qu'elle ne signifie plus rien, sinon quelques bricolages institutionnels pour assurer des fins de carrières enseignantes.

Il n'est pas de religion digne de ce nom qui ne vise à témoigner de sa possession dangereuse d'une vérité salvatrice et qui ne cherche conséquemment à la propager par tous les moyens. Beaucoup de la puissance coagulante du religieux échappe d'ailleurs à une compréhension historique ou à une description de son contenu exact. L'acte violent ne s'autorise ni ne tient à une mauvaise interprétation d'un verset ou d'une sourate.

Une religion sans martyrs, sans témoins, a la même force de persuasion qu'un descripteur officiel de cours. Cette condition nous échappe, car nous pensons le phénomène religieux dans le contexte de notre relativisme récent ou mieux à l'échelle des Chartes qui iraient, à des fins culturelles, jusqu'à autoriser peut-être le financement de son enseignement obligatoire. Plus exactement, le religieux au Québec est tombé en ruine, nous ne l'avons pas abandonné après mûre réflexion, par décision résolue. Il a été vite remplacé par une pensée molle de tolérance sans discernement, un humanisme de pacotille pour le grand nombre. Les sociétés de consommation se surprennent à croire en leurs pieux mensonges sur la nature véritable de l'être humain et arrivent à peine à imaginer un démenti cinglant. La religion n'est pas toujours un divertissement ou le moment le plus vide d'une plage horaire à l'école publique. C'est dans ce contexte que nous croyons encore que la vérité religieuse peut se présenter à l'école sous le mode pédagogique d'une "journée carrière», dans divers programmes et kiosques.

L'événement de l'effondrement des tours montre bien la nature de nos illusions quant à la finalité des religions véritables. Il commande plutôt le courage d'une société laïque, vécu sous le mode de l'affirmation de la valeur émancipatrice de la séparation stricte entre le religieux et l'État. Une religion doit se reproduire institutionnellement; elle ne saurait être pacifique sous peine de déchoir en un divertissement privé ou en une « recherche individuelle» de sens. Refuser de voir cette détermination fondamentale du religieux expose à bien des réveils brutaux. Pouvons-nous réellement prétendre, comme nous le propose l'actuelle propagande, qu'il existe de bons et de mauvais musulmans, des terroristes et des croyants ordinaires? Il subsiste des tièdes et des martyrs dans toutes les religions. L'activité religieuse n'a rien à voir avec la possession tranquille d'une vérité épanouissante, elle implique par essence une préparation pour l'avènement de son utopie fondatrice.

À cet égard, dans la vie des groupes humains la religion fait rarement partie des solutions, mais aggrave plutôt les problèmes, confirme les perceptions délirantes, conforte les haines séculaires dans une comptabilité morbide des affronts et des offenses. La religion s'établit comme 
mémoire vive et justification de la violence gratuite. Véhicule du transfert ou du transport transgénérationnel des conflits identitaires. Il faudrait pouvoir imaginer une société capable de se débarrasser progressivement - au nom même d'une éventuelle vérité religieuse - de l'inscription spatiale des lieux de culte. Le patrimoine commun de l'humanité ne saurait être à la merci d'une explosion identitaire aveugle. Démanteler ces artefacts n'est certes pas pour demain ; mais conserver vifs et enkystés des lieux de mémoire pour en subir régulièrement les retombées « radio-actives » tient du masochisme et non du respect de la différence.

La désignation de «terrorisme» appartient de fait à la catégorie de la guerre sainte. Nous faisons preuve ici d'une bien courte mémoire quant à notre propre passé inquisitorial, persécuteur et feignons de ne pas reconnaître la nature véritablement hostile des groupes humains religieusement polarisés. L'apostolat néolibéral a échoué à convertir par l'économie des populations résolues à ne pas perdre leur identité contre l'ajout de 125 canaux de télévision américains.

Il n'est pas de sociétés innocentes, surtout celles qui exécutent leurs folles progénitures en circuit fermé. Pas plus qu'il n'existe de véritables démocraties lorsqu'en leur sein des individus concentrent en un réseau des pouvoirs monétaires tels qu'ils rivalisent avec ceux de l'État de droit, au point de le diriger à distance. Nous vivons dans une ploutocratie. Loin de penser que le commerce stimule l'idéal démocratique, qu'il en serait le corollaire, la présence de géants mobiles sur la scène mondiale fait disparaître la possibilité même de l'être en commun. Pour qu'un seul homme puisse modifier son environnement, mille autres en voient le leur planifié, enserré par une discipline de servilité programmée.

Il convient plus que jamais d'interroger le vertige d'indifférence qui permettrait encore de concentrer de la vie sacrifiable, numérisable. Ne sommes-nous jamais sortis des camps, de l'assemblage dans les parcs humains ou ceux d'engraissement des sociétés de consommation avancées ? À moins qu'il ne s'agisse aujourd'hui comme hier que d'une différence de concentration des gaz à administrer à des populations civiles?

\section{Bibliographie}

BROSSAT, A. (1996). L'épreuve du désastre, Le XX $X^{e}$ siècle et les camps, Paris, Albin Michel.

DEBORD, G. (1992). Commentaires sur la société du spectacle, Paris, Gallimard.
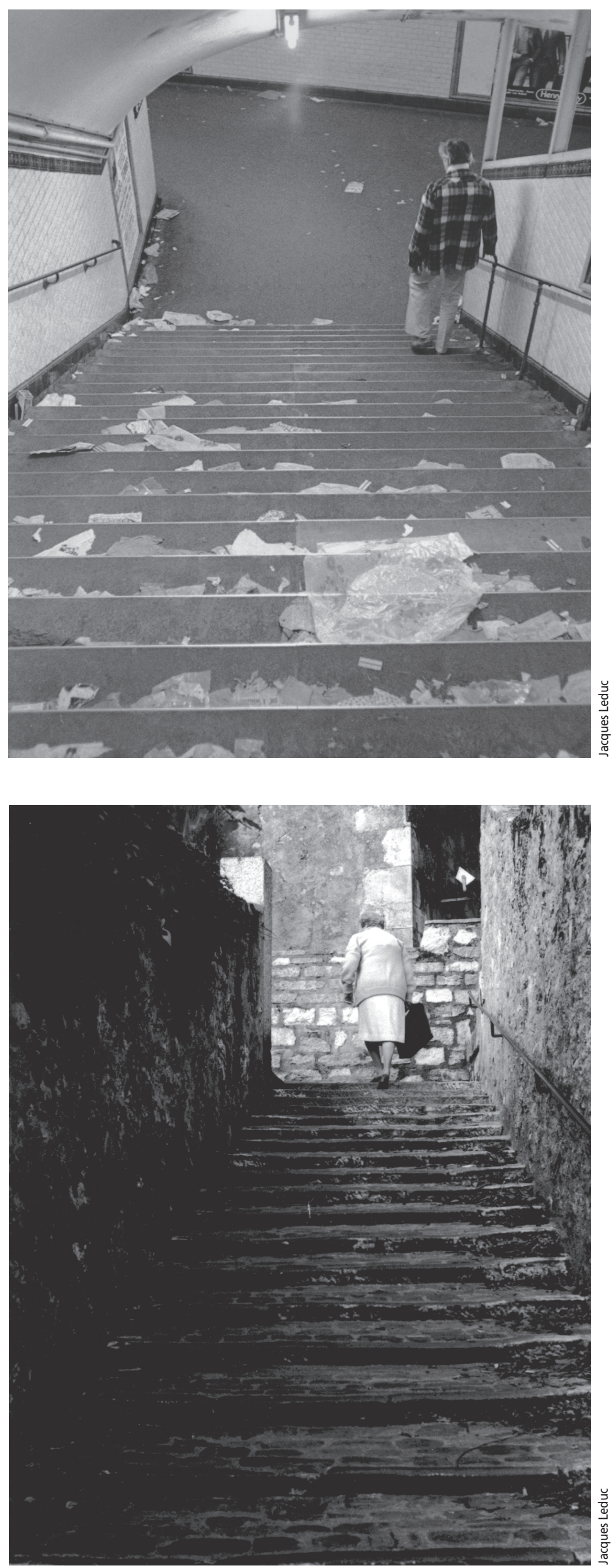